\title{
Network Modeling of Complex Dynamic Systems
}

\author{
Bosiljka Tadić \\ Department of Theoretical Physics, Jožef Stefan Institute, Ljubljana, Slovenia \\ Bosiljka.Tadic@ijs.si
}

\begin{abstract}
Networks representing complex dynamical systems often have a multiscale structure and additional properties of nodes and links, which may vary in time. We briefly summarize current trends in the statistical physics research of networks structure and dynamics and its applications to physical, biological, and social systems discussed in the Workshop.
\end{abstract}

Keywords: Networks, Complexity, Dynamics, Subgraphs.

\section{Mapping Complex Dynamical Systems to Graphs}

The idea of mapping a complex dynamical system on a graph, e.g., by identifying nodes and their connections, is to provide a ground for quantitative analysis of these systems, particularly in view of the graph theory and the statistical physics of complex networks. The approach is particularly useful in areas traditionally lacking formal theories and new emerging disciplines, specifically in the

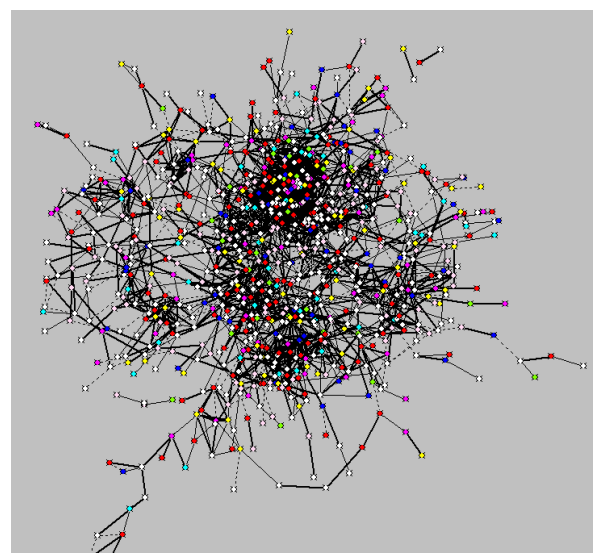

Fig. 1. Network generated from the correlations of gene expressions for the selected set of genes in genome of yeast 223. Nodes represent different genes and each color correspond to a gene function (put in 7 groups). Threshold for the correlations applied.

study of biological networks, and networks related to socio-technological interactions and economy. Science has just began to recognize the dynamically nonlinear and strongly correlated nature of these systems [1]. Furthermore, modeling 
the collective dynamics on networks [4 provides the ways towards quantitative study, scalability and universality in the emergent dynamical phenomena. Such dynamical effects play an important role in systems across different scales and nature of interactions. Two striking examples are the technology-based social communications [1] and the functional materials in nano-technology [5.

Emergent Graphs representing the complex dynamical systems both in datadriven modeling and in theoretical approaches often are sparse, scale-free, and modular. These structural properties are closely related to the systems evolution and function. Accordingly, there are constant needs to develop new/adjusted methods and algorithms to appropriately analyze and visualize these graphs.

Goals of the Workshop are to (partially) review the current works both in the theoretical and algorithmic approaches, on one side, and in the applications which primarily use methods of the statistical physics, on the other. Not of a lesser importance is a constant intention to developing communications across different disciplines and improving the converging research standards in the field.

\section{Current Trends in Network Research}

A brief summary of the research to be discussed at the Workshop includes:

- Networks from the empirical data, especially in the social sciences and bioinformatics, can be constructed in different ways (an example is shown in Fig. 1 ). Depending on the concept, a careful filtering of the data is necessary, awareness of the parameters, missing data, etc, which may affect the results.

- Topology 85 Relevant Subgraphs in multiscale networks are of great importance, particularly the subgraphs related to conserved evolutionary or functional units (communities, motifs, trees, paths, cycles) in biological networks.

- More StructureDynamics Interdependences, readily manifested in the diffusive processes on networks 44. The spin dynamics, synchronization, coupled chaotic maps, are some of the currently studied examples, where new dynamical effects occur due to the network structure and vice-versa.

- Standard $\&$ Renewed Methods, such as spectral analysis, weighted maximumlikelihood, but for different environment, M-distance matrices, reveal additional robust properties not seen immediately from the connectivity matrix.

\section{References}

1. Boccaleti, S., Latora, V., et al.: Complex Networks: Structure and Dynamics. Physics Reports 424, 175-308 (2007)

2. Cho, R.J., et al.: A Genome-Wide Transcriptional Analysis of the Mitotic Cell Cycle. Molecular Cell 2, 65-73 (1998)

3. Živkovic, J., et al.: Statistical Indicators of Collective Behavior and Functional Clusters in Gene Expression Network of Yeast. European Phys. J. B 50, 255 (2006)

4. Tadić, B., Rodgers, G.J., Thurner, S.: Transport on Complex Networks: Flow, Jamming \& Optimization. Int. J. Bifurcation and Chaos 17, 2363-2385 (2007)

5. Virtual, J.: Nanoscale Science \& Technology, http://www.vjnano.org/ 